\title{
Stand und Trends in der biologischen Rekonstruktion von Knorpelschäden des Kniegelenks
}

\author{
Bernhard Schewe, Jürgen Fritz, Christoph Gaissmaier, Kuno Weise
}

\section{Zusammenfassung}

Aufgrund seines besonderen Aufbaus fehlt dem Knorpelüberzug der Gelenkflächen im Falle eines verletzungsoder erkrankungsbedingten Defekts eine ausreichende Fähigkeit zur Selbstheilung. Da bei ausbleibender Heilung eines primär entstandenen Knorpelschadens, das sekundäre Arthroserisiko mit der Defektgröße erheblich zunimmt, ist gerade bei größeren Knorpelschäden, bestehender Symptomatik und speziell beim jüngeren $\mathrm{Pa}-$ tienten die zügige Defektsanierung angezeigt. Für die biologische Rekonstruktion von Knorpelschäden des Kniegelenks stehen heutzutage verschiedene operative Verfahren zur Verfügung. In zahlreichen tierexperimentellen und klinischen Studien konnten Differenzialindikation und Wertigkeit der einzelnen Methoden gegeneinander abgewogen und Empfehlungen zu einer individuell angepassten Vorgehensweise formuliert werden. Neben der Regeneration des zerstörten Knorpelgewebes ist die zeitgleiche Sanierung allfälliger Achsabweichungen, Meniskusschäden und/oder Bandinstabilitäten für die Wiederherstellung der Gelenkfunktion und für die weitere Prognose wichtig. Unter Berücksichtigung geeigneter Indikationskriterien sind die Ergebnisse der Knorpelzelltransplantation auch nach längeren Verlaufszeiten viel versprechend, wobei sich diese Methode auch für Defekte mit einer Größe von mehr als $4 \mathrm{~cm}^{2}$ Flächenausdehnung eignet, bei denen andere Verfahren mit biologisch rekonstruktiver Zielsetzung meist überfordert sind. Neue Ansätze und Weiterentwicklungen bestehender Methoden zur Knorpelregeneration befinden sich derzeit in der Phase experimenteller und klinischer Erprobung.

\section{Status and Trends in the Biological Reconstruction of Cartilage Injuries of the Knee}

As result of its particular construction, the cartilage covering of joint surfaces does not have a sufficient capacity for self-healing in cases of damage due to disease or injury. In the absence of healing of primary damage to cartilage, the risk of secondary arthrosis increases appreciably with the size of the cartilage defect. Thus the symptoms in cases of extensive cartilage damage, especially in young patients, are indications for a rapid defect management. Today, several operative procedures are available for the biological reconstruction of cartilage injuries of the knee joint. Numerous animal experimental and clinical studies have compared the differential indications and value of the methods and recommendations for an individually adapted procedure have been formulated. Besides regeneration of the destroyed cartilage tissue, the concomitant correction of conspicuous axis deviations, meniscus damage and/or ligament instabilities are important for re-establishing the function of the joint and for the further prognosis. Under consideration of appropriate indication criteria, the results of cartilage cell transplantation are highly promising, even after longer follow-up times. Also, this method is suitable for defects with an area of more than $4 \mathrm{~cm}^{2}$ for which other methods with the target of biological reconstruction are mostly inadequate. New approaches and further developments are currently in the stage of experimental and clinical testing.

\section{Einleitung}

Der hyaline Gelenkknorpel wird nicht durchblutet und hat keinen Anschluss an das Lymph- oder Nervensystem Seine Ernährung erfolgt ausschließlich durch Diffusion. Der synovialen Flüssigkeit, die auch als Gelenkschmiere wirkt, kommt dabei eine wichtige Funktion zu. Beim Gehen oder unter normaler Belastung wird das Knorpelgewebe komprimiert, dabei tritt Flüssigkeit aus dem Knorpel aus und Stoffwechselendprodukte werden ausgeschwemmt. Nach Entlastung saugt das Knorpelgewebe Flüssigkeit aus dem Gelenkspalt zurück. Durch diesen Vorgang werden die Knorpelzellen (Chondrozyten) mit Zuckern, Aminosäuren, Fetten und anderen Nährstoffen versorgt.

Je nach Topographie ist Gelenkknorpel unterschiedlich dick. Beim Menschen kann er im Bereich der Patella eine Schichtdicke von 7-8 mm erreichen. Die Fähigkeiten der mechanischen Dämpfung und des fast reibungslosen Gleitens der Gelenkflächen verdankt er seiner Grundsubstanz, die von den hoch spezialisierten Chondrozyten gebildet wird. Feingeweblich findet man neben Knorpelzellen, Kollagenfasern und Proteoglykane. Der Anteil der Chondrozyten macht dabei nur ca. 3\% der gesamten Knorpelmasse aus. Knorpel ist also ein zellarmes Gewebe.

Sein Matrixgerüst besteht hauptsächlich aus Kollagen Typ II, in geringen Anteilen ist auch Kollagen Typ IX, X und XI zu finden. Die Viskosität beruht auf dem hohen Gehalt an Proteoglykanen. Für Elastizität und Festigkeit sind die Kollagenfibrillen verantwortlich [14]. Trotz jahrelanger Forschung und Entwicklung ist es bis zum heutigen Tag nicht gelungen, die außergewöhnlichen Eigenschaften des hyalinen Knorpels auch nur annähernd durch einen synthetisch hergestellten Werkstoff zu ersetzen. 
Das zentrale Problem degenerativer Gelenkerkrankungen ist der zunehmende Verlust des Knorpelüberzugs der Gelenkflächen. Dieser Vorgang wird durch ein gestörtes Verhältnis zwischen Knorpel ab- und aufbauenden Stoffwechselprozessen ausgelöst. Der intensivierte Abbau nimmt dabei phasenweise den Charakter entzündlicher Ereignisse an. Das hierdurch in seinem Gleichgewicht gestörte Gewebe ist mechanisch nicht mehr belastbar und zerfällt [14].

Derzeit gültige WHO-Definition der Arthrose:

„Die Osteoarthrose ist eine nicht entzündliche Erkrankung mit entzündlichen Episoden. Sie ist eine Folge sowohl mechanischer als auch biologischer Einflüsse, die das normale Gleichgewicht zwischen Synthese und Abbau im Gelenkknorpel stören. Ausgelöst durch verschiedene Faktoren manifestiert sich das Ungleichgewicht in morphologischen, biochemischen, molekularen und biomechanischen Veränderungen. In Verbindung mit nicht systemischen, entzündlichen Episoden führen diese Vorgänge zur Erweichung, Auffaserung und zur Zerstörung der Gelenkoberfläche, sowie zum Verlust von artikulärem Knorpel und einer Sklerose des subchondralen Knochens mit Ausbildung von Osteophyten.“

\section{Umschriebene Gelenkknorpelschäden}

Als Ursache für einen umschriebenen Knorpeldefekt kommen in erster Linie traumatische Schädigungen, oft im $\mathrm{Zu}-$ sammenhang mit direkten Anpralltraumen oder Distorsionsverletzungen in Betracht. Nicht selten ist der traumatische Knorpelschaden dann nur Teil einer komplexen Gelenkbinnenverletzung. Die Ursache anderer Knorpelläsionen, wie z.B. der Osteochondrosis dissecans (OD), ist letztlich nicht vollständig geklärt. Diskutiert werden posttraumatische Knorpelveränderungen sowie Durchblutungsstörungen der subchondralen Knochenplatte $[5,6]$.

Im Gegensatz zur chronisch progressiven Erkrankung des Gelenkknorpels im Rahmen der Arthrosen stellt der traumatische Knorpelschaden eine akute Verletzung des Knorpelgewebes dar. Allerdings wird eine spontane Heilung bzw. Regeneration der „Knorpelwunde“ vor allem beim Erwachsenen nur selten beobachtet, da keine ausreichende Einwanderung und Vermehrung von Chondrozyten in den Defekt stattfindet. Kommt es allerdings bei der Verletzung des Knorpels auch zu einer Schädigung der subchondralen Knochenlamelle, entsteht ein Wundhämatom, aus dem schließlich faserknorpelartiges Reparaturgewebe entstehen kann. Aufgrund seiner untypischen molekularen und strukturellen Zusammensetzung besitzt dieses Narbengewebe jedoch nicht die hochwertigen Eigenschaften des hyalinen $\mathrm{Ge}$ lenkknorpels, was gerade bei größeren Defekten meist zum Untergang dieses Ersatzgewebes führt $[5,10]$.

\section{Diagnose}

Da der Gelenkknorpel selbst nicht innerviert ist, verursachen Knorpelschäden keine direkten Schmerzen und bleiben zunächst oft unentdeckt. Ein Knorpelschaden wird häufig erst durch das Auftreten von sekundären Symptomen klinisch auffällig: z.B. durch Gelenkblockierungen beim Ausscheren von (osteo-)chondralen Fragmenten oder durch eine schmerzhafte Entzündung der Gelenkkapsel mit Ergussbildung infolge der abnehmenden Gleitfähigkeit der Gelenkflächen. Bereits solche einfachen und allgemeinen Symptome sollten u.a. auch an einen Knorpelschaden als deren Ursache denken lassen. Der diagnostische Algorithmus unterscheidet sich ansonsten nicht von der guten unfallchirurgisch-orthopädischen Praxis.

An erster Stelle steht dabei die Anamnese und hier vor allem das Erfragen von traumatischen Ereignissen in der Vergangenheit.

Bei der körperlichen Untersuchung sollten folgende Fragen geklärt werden:

- Bestehen Achsabweichungen (Genu varum/valgum)?

- Bestehen Bewegungseinschränkungen (Streckdefizit)?

- Besteht ein Kniegelenkserguss und/ oder eine Bakerzyste?

- Ist das Gelenk bandstabil geführt?

- Können Krepitationen oder andere pathologische Geräusche ausgelöst werden?

- Besteht eine Fehlstellung (Malalignement) der Patella oder eine Luxationstendenz?

- Besteht eine Gangbildstörung und hat diese evtl. bereits zu Insertionstendopathien geführt, die nun beschwerdeführend sind?

Ein konventionelles Röntgenbild in 2 Ebenen ist auch heute noch in der Diagnostik von Gelenkknorpelschäden unverzichtbar. Auch wenn der Gelenkknor- pel selbst nicht direkt bildgebend dargestellt werden kann, so erhält man doch wertvolle Hinweise auf den Gelenkzustand. Mittels des konventionellen Röntgens sollten folgende Fragen geklärt werden:

- Besteht eine Gelenkspaltverschmälerung und ist diese medial oder lateral betont?

- Sind freie Gelenkkörper erkennbar?

- Besteht eine Chondrokalzinose?

- Bestehen bereits Osteophyten als Hinweis auf degenerative Veränderungen im Gelenk?

- Besteht ein Malalignement der Patella (defilée - Aufnahmen)?

- Besteht eine Abweichung der Beinachse (Ganzbeinaufnahme, stehend, a.-p.)?

- Existieren Hinweise auf eine rheumatoide Arthritis?

Die moderne Kernspintomographie erlaubt eine sehr detaillierte Darstellung des Gelenkknorpels, so dass auch kleinere Knorpelschäden zur Darstellung kommen. Die Kernspiontomographie sollte folgende Fragen klären:

- Sind Gelenkknorpelschäden sichtbar und wenn ja wo?

- Ist die subchondrale Platte intakt?

- Ist der subchondrale Knochen intakt? Liegt eine OD vor?

- Welche Begleitschäden sind zu erwarten (Bandrupturen, Meniskusschäden)?

Selbst wenn etliche dieser Fragen im Rahmen einer diagnostischen Arthroskopie ohnehin geklärt werden, bleibt die MR-Tomographie eine wertvolle Hilfe zur OP-Planung und erlaubt darüber hinaus auch eine Beurteilung der subchondralen Strukturen, die bei einer Arthroskopie in der Regel nicht eingesehen werden können.

Eine Kernspinuntersuchung ist im Zuge der (präoperativen) Diagnostik bei Patienten mit Verdacht auf Gelenkknorpelschäden daher in jedem Fall empfehlenswert.

Bei Verdacht auf einen Gelenkknorpelschaden oder bei anhaltenden, unklaren Kniegelenksbeschwerden ist die diagnostische Arthroskopie des Kniegelenks erforderlich [2].

Nur die Arthroskopie erlaubt eine direkte Sicht- und Tastprüfung des Gelenkknorpels. Knorpelerweichungen und partielle Knorpelablösungen können auf diese Weise erkannt werden. Die arthrosko- 
pische Untersuchung des Kniegelenks ermöglicht eine genaue Bestimmung der Größe und Qualität des Knorpelschadens und ist somit im Hinblick auf eine Differenzialdiagnose und Klassifikation des Knorpelschadens allein zielführend.

Für die Einteilung von Gelenkknorpelschäden existieren derzeit mehrere Klassifikationen, die mehr oder weniger geläufig sind. Immer noch häufig wird die Klassifikation nach Outerbridge verwendet, die vor wenigen Jahren von der International Cartilage Repair Society (ICRS) (Abb.1) aufgegriffen und nochmals differenziert wurde. Es ist mittlerweile daher empfehlenswert, umschriebene Gelenkknorpelschäden nach der ICRS-Klassifikation einzuteilen [2].

\section{Operative Therapie}

Bei Gelenkknorpelschäden, die nach dem Abschluss des Längenwachstums anhaltende Beschwerden verursachen, ist eine zügige biologische Rekonstruktion $\mathrm{zu}$ empfehlen, da ohne Ausheilung des Knorpelschadens die Ergebnisse vor allem im Langzeitverlauf und bei größe-

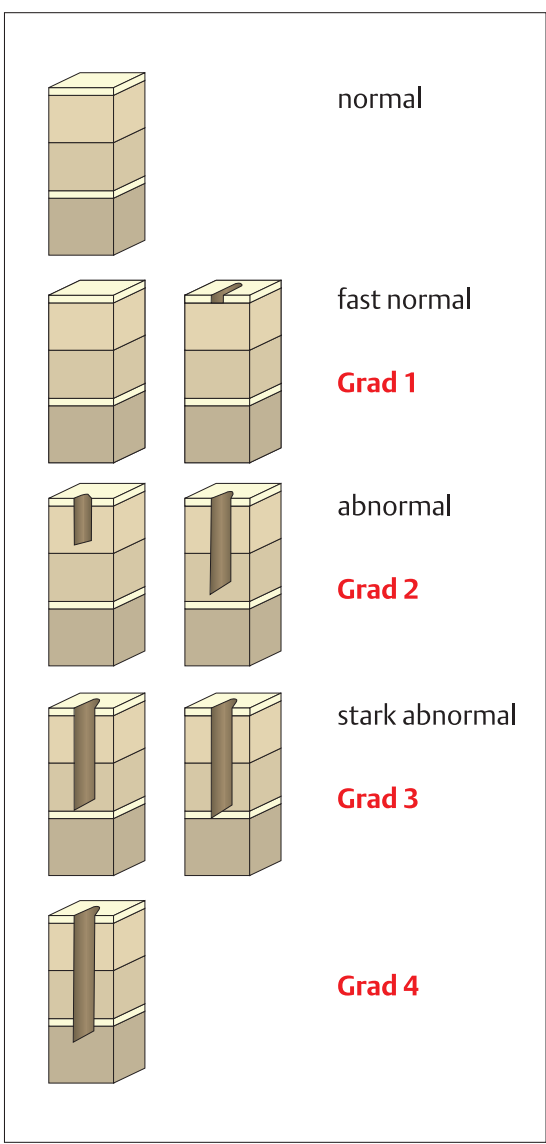

Abb. 1 Einteilung der Knorpelschäden nach ICRS. ren Defekten bedeutend schlechter sind $[2,4,5]$. Seit geraumer Zeit stehen verschiedene operative Verfahren mit biologisch rekonstruktiver Zielsetzung in der aktuellen Fachdiskussion, die teilweise bereits in prospektiv-randomisierten Studien miteinander verglichen wurden.

Verfahren wie Lavage und Debridement ermöglichen lediglich eine symptomatische Wirkung und erzielen bei vollschichtigen Knorpelschäden keine langfristige Beschwerdefreiheit oder gar eine biologische Rekonstruktion. Die Ergebnisse einer prospektiv-randomisierten und placebokontrollierten Studie sind enttäuschend [2].

Die Transplantation von Periost und Perichondrium, die reich an osteo- und chondrogenen Vorläuferzellen sind, zeigt anfänglich eine gute Wiederherstellung der Gelenkoberfläche. Im weiteren Verlauf werden jedoch häufig Verknöcherungen im Transplantat beobachtet, was zu den erheblichen Versagensquoten beider Methoden beiträgt [10]. Aufgrund der hohen Rate von Folgeeingriffen, der überwiegend schlechten klinischen Langzeitergebnisse und der zwischenzeitlichen Verfügbarkeit zuverlässigerer Methoden wird die reine Periost- und Perichondriumtransplantation nicht mehr empfohlen $[2,5]$.

Der große Vorteil der knochenmarkstimulierenden Techniken (Anbohrung Mikrofrakturierung) ist ihre arthroskopische Durchführbarkeit. Durch die Eröffnung der subchondralen Knochenlamelle wandern nach primärer Einblutung mehrheitlich unspezifische Zellen in das Defektareal ein. Es entsteht ein überwiegend fibröses Narbengewebe von verminderter Belastbarkeit [10].

Vor allem bei größeren Knorpelschäden und in Abhängigkeit der einwirkenden Belastung wird dieser Faserknorpel aufgrund seiner unzureichenden biomechanischen Eigenschaften wieder abgetragen. Dies führt häufig zu neuerlichen Beschwerden und zur weiteren Knorpeldegeneration. In einer klinischen Studie zur Mikrofrakturierung wurden die Ergebnisse bei zunächst eingetretener Verbesserung bereits nach 18 Monaten wieder signifikant schlechter [13].

Neben der Defektlokalisation und -größe ist auch das Patientenalter von Bedeutung. Im Falle einer Osteochondrosis dissecans bei noch offenen Wachstumsfu- gen sind die Ergebnisse nach Anbohrung in der Regel gut, während sie nach dem Schluss des Längenwachstums erheblich schlechter sind $[2,5]$.

Bei der im Kniegelenk häufig angewandten autologen Osteochondraltransplantation (auch als „Mosaikplastik“, „OATS“ oder „OCT“ bekannt) werden Knorpel-Knochenzylinder aus wenig belasteten Arealen des betroffenen $\mathrm{Ge}$ lenks entnommen und „press fit“ in das vorbereitete Empfängerlager des Defektbereichs - das in der Regel in der Hauptbelastungszone des Gelenks liegt - übertragen.

Neben einer Schädigung der biomechanisch wichtigen subchondralen Knochenlamelle kommt es zwischen den eingebrachten Stanzzylindern zur Bildung von Faserknorpel.

Das Ausmaß beider Effekte nimmt, neben der Inkongruenz der zu rekonstruierenden Gelenkoberfläche, mit der Zahl der übertragenen Zylinder zu [5, 9, 10].

Über die Gleitfähigkeit des so entstehenden Mischknorpels ist noch wenig bekannt, die klinischen Ergebnisse bei kleinen und mittleren Defektgrößen sind jedoch auch nach längeren Verlaufszeiten gut $[2,5,9]$. In einer prospektiv-randomisierten Studie wurden im Vergleich zur Mikrofrakturierung für die Mosaikplastik signifikant bessere Ergebnisse gefunden [8].

Dennoch spielt bei größeren Defekten zunehmend die Komorbidität der Entnahmestelle mit Beschwerden im femuropatellaren Gleitlager bei begrenzter Verfügbarkeit von Spenderzylindern eine Rolle. Außerdem wurden nach Behandlung großflächiger Knorpelschäden mit der osteochondralen Zylindertransplantation hohe Komplikations- und Revisionsraten beobachtet $[2,5]$.

Aufgrund der beschriebenen Erkenntnisse sollten die Methoden des autologen Knorpel-Knochen-Transfers bei Knorpeldefekten größer $4 \mathrm{~cm}^{2}$ daher nur in ausgewählten Einzelfällen - im Sinne eines Salvageverfahrens - eingesetzt werden $[2,5,9]$.

Für eine autologe Chondrozytentransplantation (ACT) muss zunächst ein kleines Stückchen Knorpel arthroskopisch entnommen werden. Nach 2-3 Wochen werden die hieraus angezüchteten Knorpelzellen in einer offenen Ge- 
lenkoperation unter einen zuvor über den Defekt aufgenähten Periostlappen injiziert.

In einer Studie zur ACT wurden arthroskopisch gewonnene Biopsien von $37 \mathrm{~Pa}$ tienten histologisch untersucht. In $80 \%$ der Proben wurde hyalinartiger Knorpel nachgewiesen. Das nach ACT entstehende Knorpelgewebe kann bis zu 90\% und mehr der Festigkeit des gesunden hyalinen Knorpels erreichen und ist auch in seinen biochemischen Eigenschaften nicht mit Faserknorpel zu vergleichen [15]. Ähnlich histologische Ergebnisse nach ACT wurden mittlerweile auch von einer Reihe anderer Arbeitsgruppen beschrieben.

Dass eine qualitativ hochwertige Regeneratbildung von prognostischer Bedeutung ist, lassen die Ergebnisse einer prospektiv-randomisierten Studie zur ACT vs. Mikrofrakturierung vermuten. Während in der Gruppe mit histologisch nachgewiesener Faserknorpelbildung mehrere Transplantatversager auffielen, befand sich in der Gruppe mit der Bildung eines hyalinen Regenerats auch nach längeren Verlaufszeiten kein einziger Transplantatversager $[11,12]$. In einer prospektivrandomisierten Studie zur ACT vs. Mosaikplastik wurden für die ACT teils signifikant bessere Ergebnisse gefunden [3].

Aufgrund der vorliegenden Ergebnisse zu den verschiedenen biologischen Rekonstruktionsverfahren wird die ACT daher mittlerweile von verschiedenen Autoren als Methode der Wahl bei umschriebenen Knorpeldefekten über $3-4 \mathrm{~cm}^{2}$ Defektfläche empfohlen. Die entsprechenden Empfehlungen und Leitlinien zur Indikation, Durchführung und zellbiologischen Qualitätssicherung der ACT wurden unter Berücksichtigung des derzeitigen Kenntnisstands von der Arbeitsgemeinschaft „Geweberegeneration und Gewebeersatz" der DGU und DGOOC veröffentlicht [2].

\section{Matrixgekoppelte ACT}

Die häufigste Komplikation der konventionellen ACT ist die Transplantathypertrophie, die vom aufgenähten Periostlappen ausgeht. Durch Anwendung eines geeigneten Biomaterials zur Defektabdeckung oder als Trägermaterial für die Zellen kann dieses Problem weitgehend behoben werden [4,7]. Bevor ein solches Biomaterial jedoch klinisch eingesetzt werden kann, müssen entsprechende Eignungsprüfungen durchgeführt wer- den. Auch hierzu wurden von der oben bezeichneten Arbeitsgemeinschaft der Fachgesellschaften entsprechende Vorgaben gemacht. Des Weiteren ist die $\mathrm{Zu}-$ lassung des Biomaterials für eine trägergekoppelte ACT durch die zuständigen Stellen zwingende Voraussetzung für dessen klinische Anwendung [2].

Im Vergleich zur konventionellen ACT lassen sich mit der trägergekoppelten Form Zugangswege verkleinern und Schnitt-Naht-Zeiten wesentlich reduzieren, was letztlich für den Patienten eine geringere Komorbidität und einen kürzeren stationären Aufenthalt zur Folge hat $[1,4]$. Außerdem besteht damit die Möglichkeit, die Transplantation mit weiteren notwendigen Zusatzeingriffen (Achskorrekturen, Meniskus- und Kreuzbandchirurgie) zu kombinieren.

Darüber hinaus werden derzeit auf der Grundlage bestehender und neuer Biomaterialien intelligente Trägersysteme entwickelt, die die Ergebnisse der ACT nochmals verbessern und ihr Indikationsspektrum erweitern können.

\section{Planung}

Bei der Planung einer autologen Chondrozytentransplantation (ACT) muss der Patient darüber aufgeklärt werden, dass ihm bei dieser Therapieoption zwei operative Eingriffe bevorstehen. Zunächst eine diagnostische Arthroskopie, die bei gegebener Indikation mit einer Knorpelentnahme zur Zellanzüchtung verbunden ist und ca. 2-3 Wochen später die eigentliche Chondrozytentransplantation. Das beschriebene Zeitintervall ist für die Herstellung des zellbesiedelten Implantats notwendig.

Gegebenenfalls muss der Patient auch darüber informiert werden, dass zusätzliche Eingriffe notwenig sind, die eine biologische Oberflächenrekonstruktion überhaupt erst ermöglichen. Zu den permissiven BegleitmaßnahmengehörenEingriffe am Meniskus, der Kreuzbandersatz und die Korrektur einer Achsfehlstellung. Sinn und Zweck dieser Eingriffe ist die Wiederherstellung einer möglichst physiologischen Biomechanik des Gelenks.

Der Patient muss über die Notwendigkeit von zwei Eingriffen für die Knorpelzelltransplantation aufgeklärt werden. Außerdem muss auf die Notwendigkeit von Zusatzeingriffen wie Achskorrektur, Meniskus- und Kreuzbandchirurgie hingewiesen werden.
Folgende Grundvoraussetzungen für eine ACT sollten gegeben sein:

- vollschichtige Knorpeldefekte, die größer als ca. $3 \mathrm{~cm}^{2}$ sind

- Osteochondrosis dissecans Stadium III und IV mit einem Defekt größer als ca. $3 \mathrm{~cm}^{2}$ (dabei ist in den meisten Fällen eine Spongiosaplastik aus dem Beckenkamm erforderlich, bei kleineren Defekten kann die Spongiosa auch aus der Femurkondyle entnommen werden)

- tragfähiger Umgebungsknorpel

- intakte korrespondierende Gelenkfläche (Schädigung bis maximal Grad II nach ICRS-Klassifikation ist zulässig)

- intakter Meniskus (Teilresektion bis max. ein Drittel des Gesamtvolumens ist zulässig)

- maximal zwei unabhängige Defekte, die nicht korrespondieren (keine „kissing lesions")

- intakte Bandführung, physiologische oder korrigierte Beinachse

- freie Gelenkbeweglichkeit

Kontraindikationen

Von einer ACT sollte abgesehen werden bei:

- Osteoarthrose

- Gelenksteife

- Arthrofibrose

- Abweichungen von der physiol. Beinachse (Korrektur vor oder während $\operatorname{der} \mathrm{ACT}$ )

- total/subtotal reseziertem Meniskus

- insuffizienter Bandführung (Korrektur vor oder während der ACT)

- Patellamalalignement (Korrektur vor oder während der ACT)

- implantierten Carbonstiften

- entzündlichen Gelenkerkrankungen (z. B. rheumatoide Arthritis)

Weitere Ausschlusskriterien für die ACT

- chronische Infektionskrankheiten

- Tumorkrankheiten

- metabolische Arthropathien (z. B. Gicht/Pseudogicht)

- autoimmunologische Erkrankungen

- Borreliose

- schwere neurologische Erkrankungen

- Adipositas (Body-Mass-Index > 35)

- Schwangerschaft

- Suchterkrankungen

- psychische Erkrankungen mit reduzierter Compliance

\section{Technik}

Im Rahmen einer diagnostischen $\mathrm{Ar}$ throskopie muss geklärt werden, ob eine biologische Knorpelrekonstruktion überhaupt erforderlich ist. Liegt ein sanierungsbedürftiger Knorpelschaden vor, 


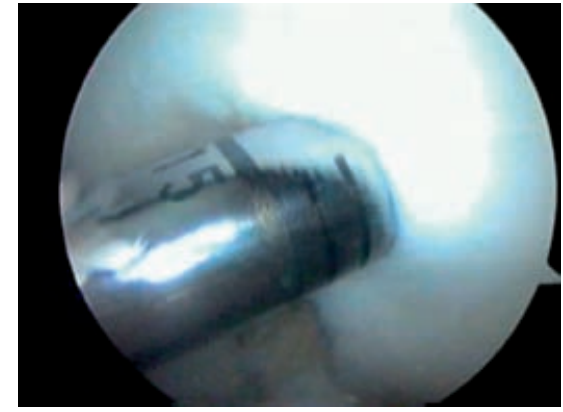

Abb.2 Entnahme eines Knorpel-Knochenzylinders mit einer Hohlstanze an der Notchkante.

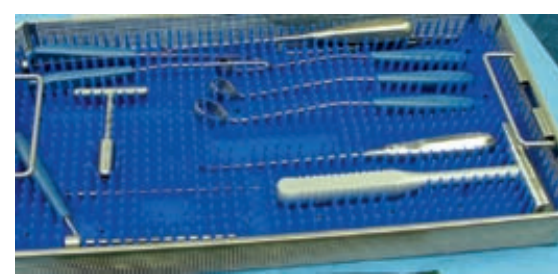

Abb.3 Transplantationsset mit konfektionierten Stanzen.

muss geprüft werden, ob eine biologische Rekonstruktion möglich ist (Ausschluss von Kontraindikationen) und welches Rekonstruktionsverfahren für die jeweilige Defektsituation am besten geeignet ist (z.B. Mikrofrakturierung, Knorpel-Knochen-Transplantation oder ACT). Zur Klärung dieser wichtigen Fragen sollte die Ausdehnung und Tiefe des Defekts genau bestimmt werden, ein Tasthaken mit Größenskalierung ist hierbei hilfreich. Außerdem muss der umgebende Knorpel untersucht und mögliche Begleitpathologien, wie Meniskusläsionen oder eine Kreuzbandinsuffizienz, verifiziert werden. Erst die genaue Kenntnis der beschriebenen Einflussgrößen ermöglicht das differenzierte therapeutische Vorgehen.

Zur Knorpelzellzüchtung werden abhängig von der Größe des zu behandelnden Defekts 2-3 Knorpelzylinder benötigt. Die Entnahme von Knorpel-KnochenZylindern ermöglicht die Zellisolation aus hierfür besonders geeigneten Knorpelschichten (Abb.2). Ziel ist die Anzucht von biologisch möglichst hochwertigen Chondrozyten unter Verwendung standardisierter und validierter Verfahren.

Zur Knorpelzüchtung werden je nach Defektgröße 2-3 Knorpelzylinder benötigt. Dabei ermöglicht die Entnahme von Knorpel-Knochen-Zylindern die Zellisolation aus hierfür besonders geeigneten Knorpelschichten.
$\mathrm{Zu}$ empfehlen ist die Verwendung von geeigneten Hohlstanzen mit einem Durchmesser von $4 \mathrm{~mm}$, wie sie in ähnlicher Form auch für die Knorpel-Knochen-Transplantation verwendet werden. Eine geeignete anatomische Lokalisation zur Entnahme der KnorpelKnochen-Stanzen ist die mediale oder laterale Kante der Notch. Hier findet sich eine ausreichend dicke Knorpelschicht in einer nicht belasteten Gelenkregion. Außerdem ist diese Region über die Standardzugänge gut erreichbar.

Die entnommenen Zylinder werden in ein steriles Gefäß mit Nährmedium abgeworfen, sofort verschlossen und in einem speziellen Transportbehälter zur Weiterverarbeitung an den Hersteller des Transplantats versandt.

Die Transplantation erfolgt über eine Arthrotomie, die parapatellar angelegt wird. Die Arthrotomie sollte dabei einen möglichst direkten Zugang zum Defekt ermöglichen. Je nach Lokalisation des Defekts sollte an der medialen oder lateralen Femurkondyle eine parapatellar mediale oder laterale Schnittführung gewählt werden.

Bei einer retropatellaren Lokalisation ist ein parapatellarer lateraler Zugang zu empfehlen, da von lateral die Patellarückfläche durch Aufstellen der Kniescheibe besser zu erreichen ist. Außerdem kann dabei ein laterales Release durchgeführt werden. Dies ist häufig für die Entlastung der retropatellaren $\mathrm{Ge}$ lenkfläche notwendig.

Der zu behandelnde Defekt wird dann durch entsprechende Lagerung des Beins in Beugung so eingestellt, dass er gut über eine Miniarthrotomie erreicht werden kann.

Zunächst wird zerstörtes Knorpelgewebe oder minderwertiges Narbengewebe entfernt. Zur technischen Vereinfachung können konfektionierte Stanzen in verschiedenen Größen verwendet werden (Abb.3). Alternativ kann der Defekt mit einem Skalpell umschnitten werden.
Wichtig ist dabei, dass die Resektion sparsam im gesunden Gewebe, aber mit ausreichendem Abstand zum Defekt erfolgt, damit um die Läsion eine stabile Knorpelschulter entsteht. Beim Entfernen des Knorpels sollte die subchondrale Knochenlamelle intakt bleiben, die Zone des mineralisierten Knorpels sollte jedoch unbedingt entfernt werden. Blutungen aus der subchondralen Knochenlamelle sind möglichst zu vermeiden. Kleinere punktuelle Blutungen können mit einem Tropfen Fibrinkleber gestillt werden.

Defekte in der subchondralen Knochenlamelle sollten mit Spongiosa aufgefüllt werden. Dazu eignen sich Spongiosazylinder, die mit dem Instrumentarium für die Knorpel-Knochen-Transplantation gewonnen werden können. Als Entnahmeort empfiehlt sich aufgrund der Spongiosaqualität der ipsilaterale Beckenkamm. Bei kleineren Knochendefekten kann auch Spongiosa aus der betroffenen Femurkondyle verwendet werden. Gewonnene Zylinder können wie bei der Technik der Knorpel-Knochen-Transplantation press-fit eingebracht werden (Abb.4). Unerwünschte Blutungen aus dem Knochenlager werden dadurch in der Regel verhindert.

Nach Abschluss des Debridements muss die subchondrale Knochenlamelle frei von Knorpelresten sein. Bei der Anwendung der konventionellen ACT mit Periostlappenabdeckung oder einem Gel als Trägermaterial sollte eine stabile Knorpelschulter zum gesunden Umgebungsknorpel bestehen. Im Gegensatz hierzu ist bei der Transplantation einer geeigneten soliden Matrix, (z.B. NOVOCART ${ }^{\circledR}$ 3D, Fa. Aesculap, Tuttlingen) eine zirkulär intakte Knorpelschulter nicht zwingend erforderlich. Unter diesen Bedingungen ist eine punktuelle Fixierung des Trägermaterials ausreichend.

Für die passgenaue Applikation wird aus der zellbesiedelten Matrix ein zum Defekt kongruentes Stück ausgeschnitten. Dies kann unter Verwendung derselben Stanze erfolgen, mit der bereits der Defekt präpariert wurde (Abb.5). Alternativ hierzu kann aus einem Fadenpäckchen ein „Schnittmuster“ des Defekts angefertigt werden und damit ein entsprechend größenangepasstes Implantat aus der Matrix ausschneiden zu können.

Die Fixierung der Matrix im Defekt erfolgt mit Einzelknopfnähten (Abb.6). Besonders geeignet ist monofiles Fadenma- 


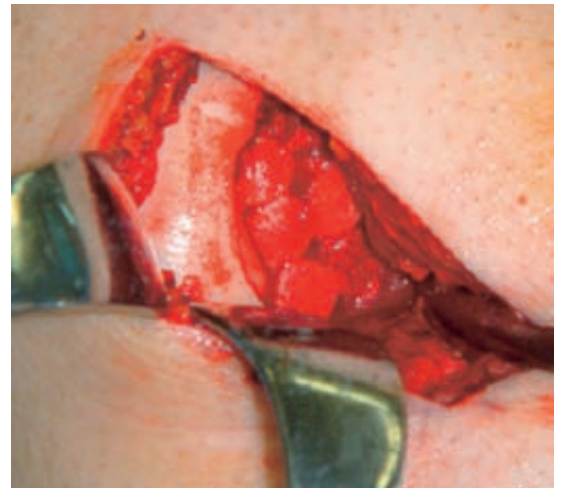

Abb.4 Auffüllen eines Knochendefekts mit Knochenzylinder aus dem Beckenkamm.

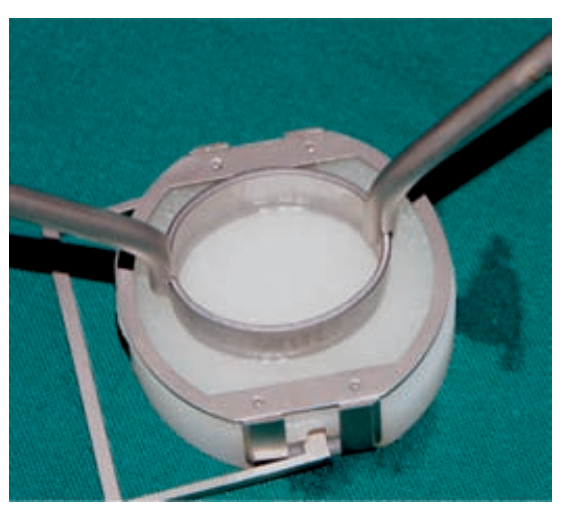

Abb.5 Ausstanzen eines kongruenten Matrixläppchens mit einer konfektionierten Stanze, mit der zuvor der Defekt ausgestanzt wurde.

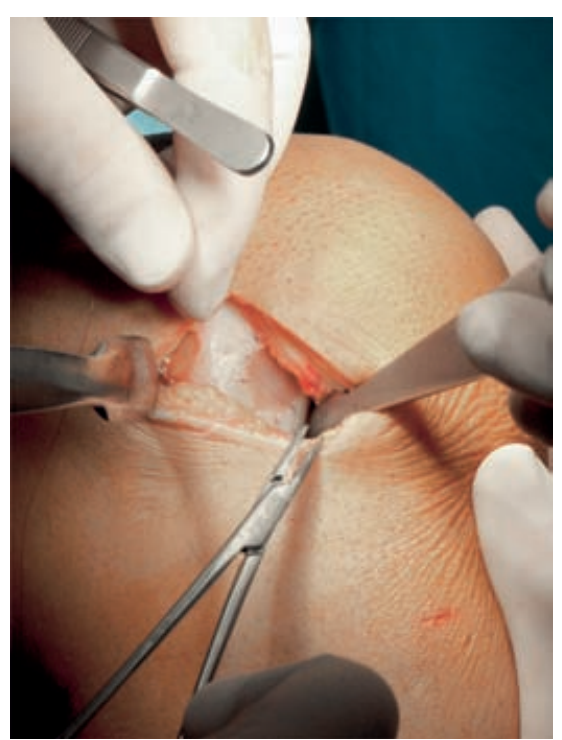

Abb.6 Einnähen der Matrix mit Einzelknopfnähten.

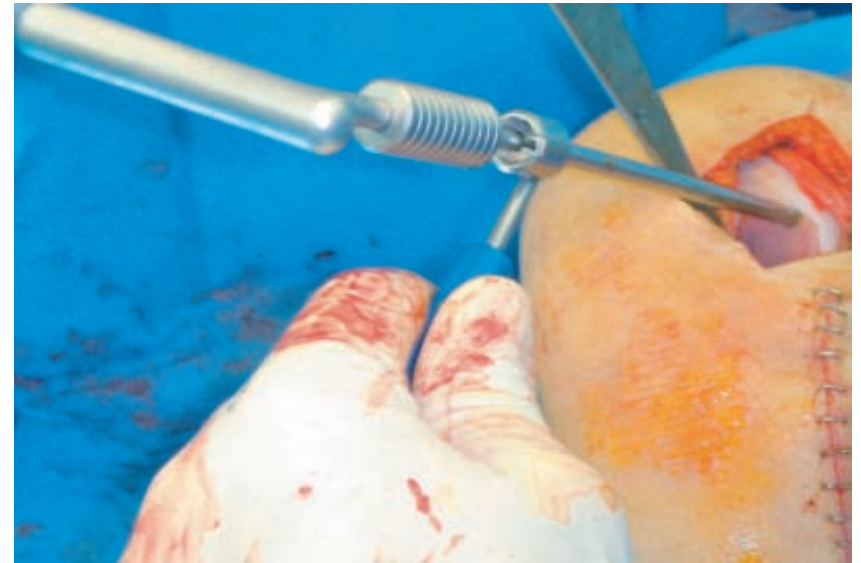

Abb.7 Alternativ besteht die Möglichkeit, die Matrix mit resorbierbaren Pins zu fixieren.

terial der Stärke 6/0. Alternativ dazu besteht die Möglichkeit, die Matrix mit kleinen resorbierbaren Minipins zu fixieren (Abb. 7).

Dazu wird eine Führungshülse mit kleinen Haltespitzen direkt randständig auf die oberflächliche Membran der Matrix aufgesetzt. Mit dem entsprechenden Pfriem werden dann die Matrix und die subchondrale Knochenlamelle perforiert. Anschließend wird der Pin mit einem dünnen Trokar in die vorgebohrte Öffnung eingebracht. In der Regel genügen 4-6 Einzelknoten oder Pins zur sicheren Fixierung der Matrix.

Fibrinkleber sollte nicht in direktem Kontakt zwischen Implantat und subchondraler Knochenplatte verwendet werden, da Hinweise bestehen, dass konventioneller Fibrinkleber mit seiner hohen Proteindichte das Anwachsen der transplantierten Knorpelzellen auf der Knochenlamelle stört.

Bei der Fixierung ist darauf zu achten, dass das Transplantat nicht unter zu starke Spannung gebracht wird. Des Weiteren sollte man sich vor der endgültigen Fixierung vergewissern, dass keine relevanten Blutungen aus der subchondralen Knochenplatte bestehen, die $\mathrm{zu}$ einem Abheben des Transplantats aus dem Defekt führen könnten.

Abschließend sollte das Gelenk vorsichtig durchbewegt werden, um zu prüfen, ob das Transplantat ausreichend stabil im Defekt einliegt.

Nach vorsichtiger Spülung erfolgt die Einlage einer Redondrainage. Die Drainage sollte dabei ohne Sog, als Überlaufdrainage, eingebracht werden. Anschlie- ßend wird die Wunde schichtweise verschlossen. Die Redondrainage sollte am 2. postoperativen Tag entfernt werden. Mit physiotherapeutischer Nachbehandlung wird ab dem 2. postoperativen Tag begonnen.

\section{Nachbehandlung}

Für die Nachbehandlung ist die Lokalisation der durchgeführten Transplantation entscheidend. Bei Transplantationen an den Kondylen darf sofort postoperativ mit Bewegungsübungen ohne Limitierung begonnen werden. Der postoperative Einsatz einer Motorschiene ist $\mathrm{zu}$ empfehlen. Die Mobilisierung muss mit Teilbelastung erfolgen. In den ersten 6 Wochen sind $20 \mathrm{~kg}$ Teilbelastung einzuhalten. Ab der 7. postoperativen Woche kann die Belastung alle 2 Wochen um $20 \mathrm{~kg}$ bis zur Vollbelastung gesteigert werden.

Bei der Versorgung retropatellarer Defekte wird die Beugung postoperativ auf $60^{\circ}$ limitiert. Nach der 6 . Woche darf die Beugung alle 2 Wochen um $20^{\circ}$ gesteigert werden, bis hin zur freien Beweglichkeit. Das Bein darf dabei in Streckstellung vollbelastet werden. Zum Schutz vor unbeabsichtigter, vermehrter Beugung sollte anfangs eine bewegungseinschränkende Schiene getragen werden.

Nach Erreichen der Vollbelastung bzw. der freien Beweglichkeit sollte die kniegelenksstabilisierende Muskulatur auftrainiert werden.

Nach 6 Monaten darf Laufen auf ebenem Grund, Schwimmen und Radfahren durchgeführt werden. Nach 12 Monaten können auch Kontaktsportarten wieder aufgenommen werden. 


\section{Fazit für die Praxis}

Aufgrund der beträchtlichen Zahl umschriebener Knorpeldefekte im Kniegelenk bei Erwachsenen und Jugendlichen sowie der in zahlreichen Publikationen beschriebenen Folgeerscheinungen in unbehandelten Fällen wird eine situationsangepasste, zeitnahe Sanierung der Gelenkoberfläche empfohlen. Hierfür stehen verschiedene Verfahren mit biologisch rekonstruktiver Zielsetzung zur Verfügung, deren jeweiliger Einsatz auf die Bedingungen des Individualfalls abzustimmen ist. Ziel des ausgewählten Operationsverfahrens ist die Wiederherstellung einer möglichst optimalen Lastund Druckverteilung zwischen den Gelenkflächen. Komplementär dazu muss auch die Sanierung allfälliger Zusatzläsionen, wie Achsenfehlstellungen bzw. Meniskus- und/oder Bandschäden, in das therapeutische Kalkül einbezogen werden.

Während sich die Mikrofrakturierung zur Behandlung kleinerer Knorpelschäden von 2 bis maximal $3 \mathrm{~cm}^{2}$ nicht zuletzt bei Kindern und Jugendlichen bewährt hat, wobei grundsätzlich ein gesunder Umgebungsknorpel vorhanden sein sollte, können mit der autologen Osteochondraltransplantation (OCT) Defekte bis zu einer Ausdehnung von 3$4 \mathrm{~cm}^{2}$ angegangen werden.

Ausgedehnte Knorpelschäden bis max. $15 \mathrm{~cm}^{2}$ bei wiederum gesundem Umgebungsknorpel sind eine Indikation für die autologe Knorpelzelltransplantation. Dieses Verfahren ist jüngeren Patienten bis zu einem Lebensalter von ca. 50 Jahren und solchen Situationen vorbehalten, in denen die angrenzende Knorpelfläche intakt ist.

Das zweizeitige Verfahren umfasst die arthroskopische Entnahme zweier kleiner Knorpel-Knochen-Zylinder aus unbelasteten Randbereichen des Gelenkes, deren knorpeliger Anteil im Labor bearbeitet wird, indem man durch Isolierung der Chondrozyten sowie deren Kultivierung nach bestimmten Kriterien innerhalb von 2-3 Wochen die Anzahl der Knorpelzellen auf mehrere Millionen erhöhen kann.
Während das klassische Verfahren der ACT für den Zweiteingriff die Präparation und das Einnähen eines Periostlappens erforderlich macht, um darunter die Knorpelzell-Suspension injizieren zu können, ist das Verfahren mit der matrixgekoppelten Transplantation zeitlich weniger aufwändig und kann über eine deutlich kürzere Inzision sowie mit einer geringeren Operationsbelastung durchgeführt werden.

Die bisherigen Ergebnisse dieser Technik zeigen ein ganz überwiegend positives Outcome, wobei der hyalinähnliche Knorpelersatz bezüglich seiner mechanischen Eigenschaften mit zunehmender Zeitdauer an Stabilität gewinnt.

In Zukunft werden mutmaßlich neue biologische Rekonstruktionsverfahren entwickelt werden, die sich mit der Anwendung von Stammzellen und mit weiteren Verfahren des Tissue Engineerings befassen. Ziel ist es, durch eine möglichst optimale Wiederherstellung der Knorpelflächen des Kniegelenks eine Arthrose und damit Operationsverfahren wie den endoprothetischen Ersatz vermeiden zu können.

\section{Literatur}

1 Behrens P, Bitter T, Kurz B, Russlies M. Matrix-associated autologous chondrocyte transplantation/implantation (MACT/MACI)5-year follow-up. Knee 21 [Epub ahead of print] 2006

2 Behrens P, Bosch U, Bruns J, Erggelet C, et al. Indikations- und Durchführungsempfehlungen der Arbeitsgemeinschaft „Geweberegeneration und Gewebeersatz" der DGU \& DGOOC zur Autologen Chondrozyten-Transplantation (ACT). Z Orthop Ihre Grenzgeb 2004; 142: 529-539

3 Bentley G, Biant LC, Carrington RW, et al. A prospective randomised comparison of autologous chondrocyte implantation versus mosaicplasty for osteochondral defects in the knee. J Bone Joint Surg Br 2003; 85: 223 230

4 Fritz J, Gaissmaier C, Schewe B, Weise K. Stellenwert und Technik der Autologen Chondrozyten-Transplantation. Zentralbl Chir 2005; 130: 327-332

5 Gaissmaier C, Fritz J, Mollenhauer J, et al. Verlauf klinisch symptomatischer Knorpelschäden des Kniegelenks: Ergebnisse ohne und mit biologischer Rekonstruktion. Dtsch Arztebl 2003; 100: A 2448-2453

6 Gaissmaier C, Fritz J, Schewe B, Weise K, Mollenhauer J, Aicher WK. Cartilage Defects: Epidemiology and Natural History. Osteosynthesis and Trauma (in press) 2006
7 Gooding CR, Bartlett W, Bentley G, Skinner JA, Carrington R, Flanagan A. A prospective, randomised study comparing two techniques of autologous chondrocyte implantation for osteochondral defects in the knee: Periosteum covered versus type I/III collagen covered. Knee 24 [Epub ahead of print] 2006

8 Gudas R, Kalesinskas RJ, Kimtys V, et al. A prospective randomized clinical study of mosaic osteochondral autologous transplantation versus microfracture for the treatment of osteochondral defects in the knee joint in young athletes. Arthroscopy 2005; 21: 1066-1075

${ }^{9}$ Hangody L, Fules P. Autologous osteochondral mosaicplasty for the treatment of fullthickness defects of weight-bearing joints: ten years of experimental and clinical experience. J Bone Joint Surg Am 85 2003; (Suppl 2): 25-32

10 Hunziker EB. Articular cartilage repair: basic science and clinical progress. A review of the current status and prospects. Osteoarthritis Cartilage 2002; 10: 432-463

11 Knutsen G, Drogset JO, Engebretsen L, et al. Autologous chondrocyte implantation compared with microfracture in the knee (five year follow up). 6th Symposium of the International Cartilage Repair Society. San Diego, CA, USA, January 8-11 2006

12 Knutsen G, Engebretsen L, Ludvigsen TC, et al. Autologous chondrocyte implantation compared with microfracture in the knee - a randomized trial. J Bone Joint Surg Am 2004; 86: 455-464

13 Kreuz PC, Steinwachs MR, Erggelet C, et al. Results after microfracture of full-thickness chondral defects in different compartments in the knee. Osteoarthritis Cartilage [Epub ahead of print] 2006

14 Otte P. Physiologie der Gelenkerhaltung. In: Otte P, Hrsg.: Der Arthrose-Prozess. Gelenkerhaltung - Gefährdung - Destruktion. Teil 1: Osteochondrale Strukturen. Novartis Pharma, Nürnberg 2000; 7-1 92

15 Peterson L, Brittberg M, Kiviranta I, Akerlund EL, Lindahl A. Autologous chondrocyte transplantation. Biomechanics and long-term durability. Am J Sports Med 2002; 30: 2-12

Dr. med. Bernhard Schewe

Oberarzt

Dr. med. Jürgen Fritz

Assistenzarzt

Dr. med. Christoph Gaissmaier

Assistenzarzt

Prof. Dr. med. Kuno Weise

Ärztlicher Direktor

BG-Unfallklinik Tübingen

Schnarrenbergstr. 95

72076 Tübingen 